\title{
Editorial
}

\section{Theory and Applications of Complex Networks 2014}

\author{
Tingwen Huang, ${ }^{1}$ Qinggang Meng, ${ }^{2}$ Zhichun Yang, ${ }^{3}$ Chuandong Li, ${ }^{4}$ He Huang, \\ Jianguo Du, ${ }^{6}$ and Wei Zhang ${ }^{7}$
}

${ }^{1}$ Science Program, Texas A\&M University at Qatar, P.O. Box 23874, Doha, Qatar

${ }^{2}$ Department of Computer Science, Loughborough University, Loughborough, Leicestershire LE11 3TU, UK

${ }^{3}$ College of Mathematics Sciences, Chongqing Normal University, Chongqing, China

${ }^{4}$ School of Electronics and Information Engineering, Southwest University, Chongqing 400715, China

${ }^{5}$ School of Electronics and Information Engineering, Soochow University, Suzhou 215006, China

${ }^{6}$ School of Management, Jiangsu University, Zhenjiang 212013, China

${ }^{7}$ College of Computer Science, Chongqing University, Chongqing 400044, China

Correspondence should be addressed to Tingwen Huang; tingwen.huang@qatar.tamu.edu

Received 18 December 2014; Accepted 18 December 2014

Copyright (C) 2015 Tingwen Huang et al. This is an open access article distributed under the Creative Commons Attribution License, which permits unrestricted use, distribution, and reproduction in any medium, provided the original work is properly cited.

The purpose of this special issue is to track the latest process of mathematical and computational analysis, engineering and applications, and others. It is well known that mathematical and computational analysis as a hot topic will promote the development of society in different fields, such as complex networks, neural networks, evolution computation, and researches of optimization and algorithm. Therefore, it is meaningful to investigate the new theory, mythology, and method for the engineering and applications.

We receive many high-quality submissions that have been included in this special issue. These articles cover theoretical contributions and applications in project delivery method and energy-efficient scheduling. A brief summary of these articles is provided below.

Firstly, we are focused on mathematical and computational analysis of nonlinear dynamic networks. In the article "Passivity Analysis of Complex Delayed Dynamical Networks with Output Coupling" by Y.-F. Kang et al., constructing new Lyapunov functional, some sufficient conditions ensuring the input passivity and output passivity are obtained. H. Zhu and C. Feng studied existence and global uniform asymptotic stability of pseudo almost periodic solutions for CohenGrossberg neural networks with discrete and distributed delays. J.-E. Zhang investigated combination-combination hyperchaos synchronization of complex memristor oscillator system. T. Kerh et al. investigate nonlinear shoreline multiperiod change from orthophoto map information by using a neural networks model. C. Li et al. extend the three-dimensional SIR model to four-dimensional case and then analyze its dynamical behavior including stability and bifurcation. J.-A. Wang and X.-Y. Wen investigated sampleddata synchronization for complex dynamical networks with time-varying coupling delay and random coupling strengths.

Secondly, we are focused on engineering. The article "Energy-Efficient Scheduling for Tasks with Deadline in Virtualized Environments" by G. Du et al. proposed a novel scheduling algorithm for heterogeneous virtual machines in virtualized environments to effectively reduce energy consumption and finish all tasks before a deadline. They seek to implement an energy-efficient task scheduling algorithm for virtual machines with changeless speed comprising two main steps: assigning as many tasks as possible to virtual machines with lower energy consumption and keeping the makespan of each virtual machine within a deadline. In addition, the new scheduling strategy is simulated using the CloudSim toolkit package. Q. Huang et al. proposed a fractional-order scalar controller and its circuit implementation control for fractional-order Chen chaotic system. X. Wang et al. proposed to fit the multicast need of wireless mesh network based on the MAODV multicast routing protocol of ad hoc network, and a multicast routing protocol DT-MAODV (dynamic topology MAODV) is proposed. 
C. Chen et al. proposed the resource allocation problem by the conception of Pareto optimization, a multiobjective optimization approach. C. Ren et al. studied the method of INS auxiliary based on neural network improving the dynamic characteristics of GPS carrier tracking loop. In the article "Computational Experiment Study on Selection Mechanism of Project Delivery Method Based on Complex Factors," X. Ding et al. propose a new project delivery method by using the project owner (or project manager) for organizing design, construction, and other operations in a construction project.

The third section is focused on applications. D. Yang and $\mathrm{X}$. Zhao aimed at studying the evolution pattern of cultivating the ability of university students' entrepreneurship; this paper established the payoff matrix between the university and students agents with the evolutionary economics method. N. Shen et al. studied the influence of grinding conditions on resulting surface residual stresses in grinding titanium alloy TC4. In the article "Interactive High Quality Video Streaming via Coequal Support Video Transcoder," S. Vetrivel and G. Athisha proposed a problem in video transcoding, which is by giving a target bit rate, to determine at spatial resolution. The spatial resolution, chronological resolution, and amplitude classically were proscribed by the quantization step size (QS) to code the video. Y. Li et al. built a model for present earning value of water treatment plants in planning periods, aiming at the influence of water supply radius on present earning value. In consideration of the influence on annual earnings of water treatment plants within planning periods caused by the investment in affiliated pipe network of water treatment plants, this paper adopts the methods of minimal spanning tree and Lagrange's undetermined multiplier to optimize the pipe network within water supply areas of water treatment plants and then determines the head loss of the optimal pipe section and economical pipe diameter.

In summary, all of these papers presented recent developments, with a focus on mathematical and computational analysis, engineering and applications, information processing, modeling, and control using computational intelligence. These papers presented the latest researches in a coherent way. In this editorial, we artificially categorize them into the above to help readers to understand the organization of this special issue. We hope the reader will share our joy and find this special issue very useful.

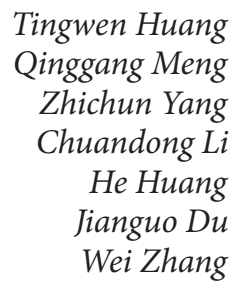




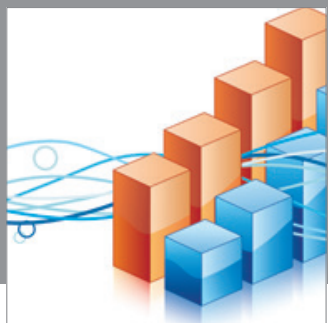

Advances in

Operations Research

mansans

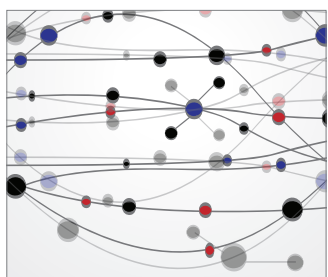

The Scientific World Journal
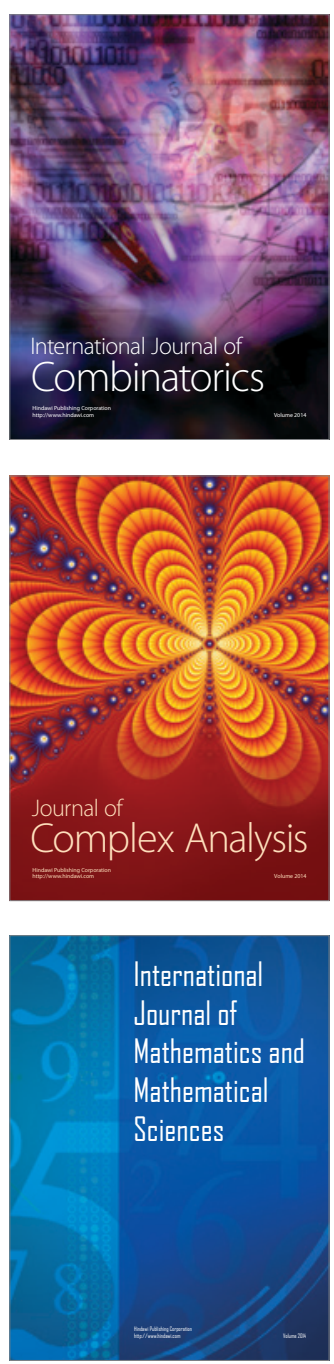
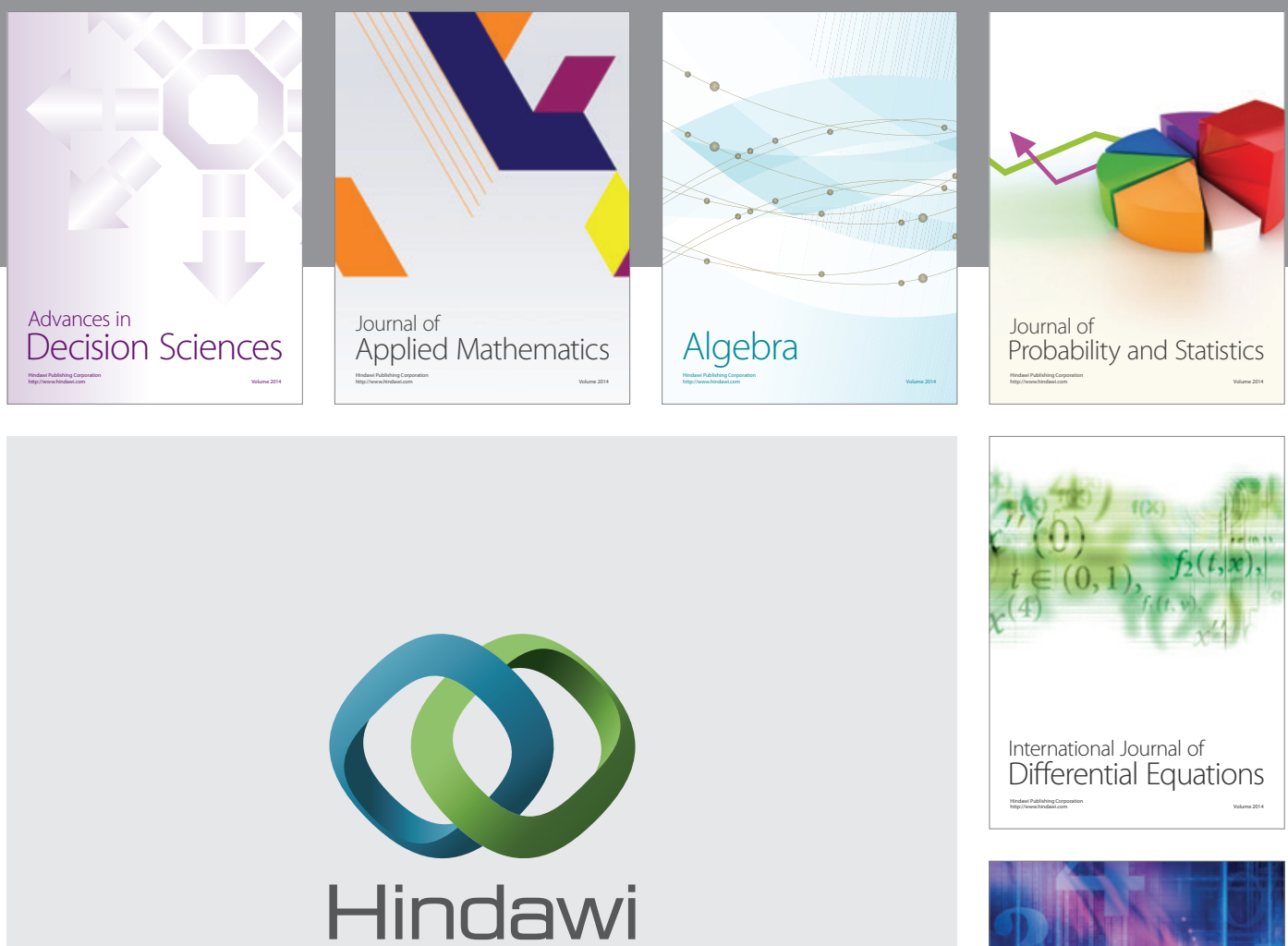

Submit your manuscripts at http://www.hindawi.com
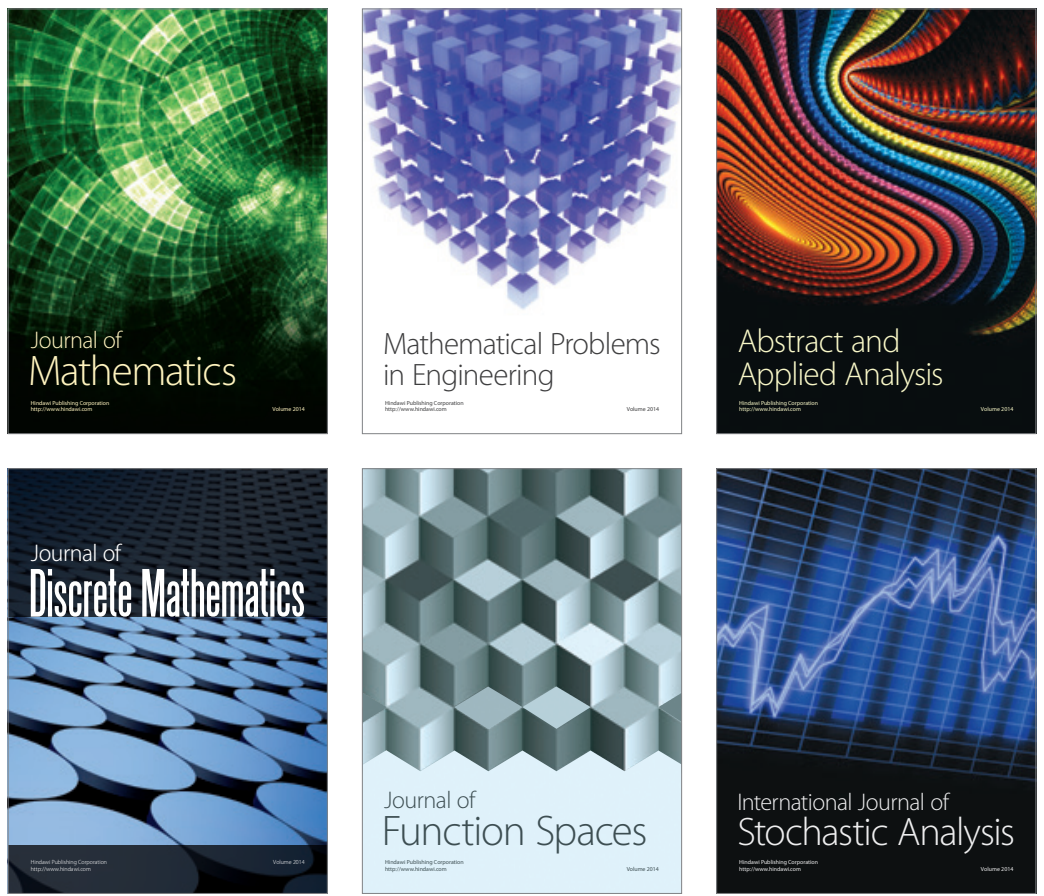

Journal of

Function Spaces

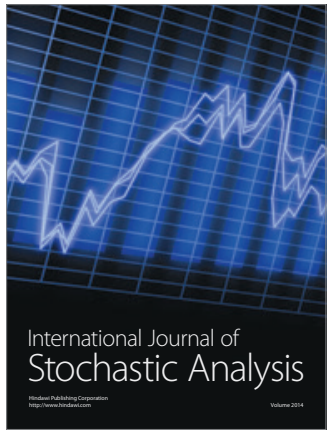

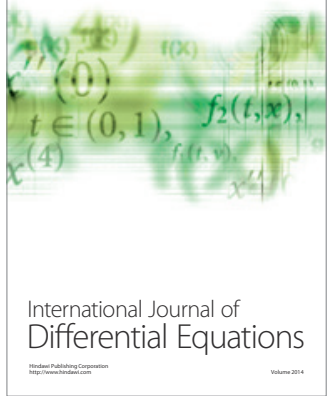
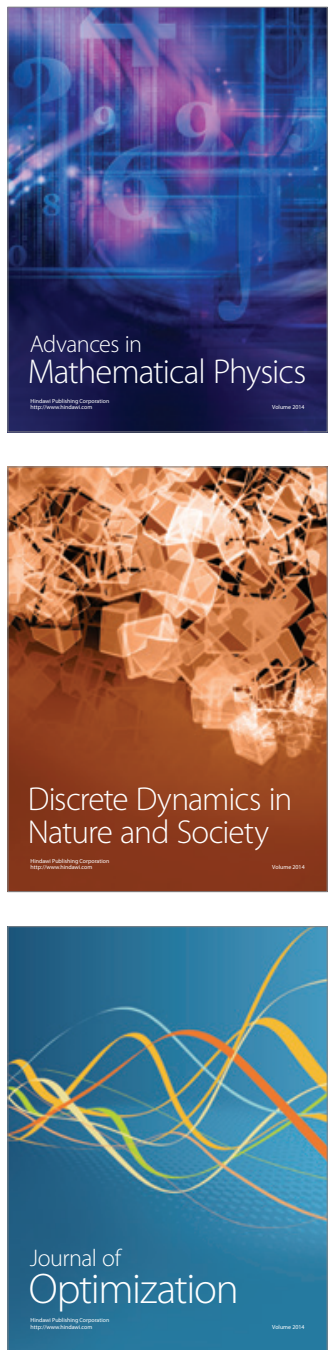